\title{
Bacteraemia with Cedecea neteri in a patient with systemic lupus erythematosus
}

\author{
A Aguilera, J Pascual, E Loza, J Lopez, G Garcia, F Liaño, C Quereda, J Ortuño
}

\begin{abstract}
Summary
We describe a case of Cedecea neteri bacteraemia in a patient with systemic lupus erythematosus during an acute severe exacerbation of the disease, which led to her death.
\end{abstract}

Keywords: Cedecea neteri, systemic lupus erythematosus

\section{Introduction}

Enterobacteriaceae of the genus Cedecea have rarely been isolated from human clinical specimens, ${ }^{1,2}$ and true bacteraemia has been documented on only one previous occasion. ${ }^{2} \mathrm{We}$ report on a patient with systemic lupus erythematosus (SLE) who developed an acute flare-up of the disease with bacteremia due to Cedecea neteri which led to her death.

\section{Case report}

A 27-year-old female patient was diagnosed in 1983 as having SLE with dermatological arthritic and serological criteria. She was managed with prednisone plus azathioprine during the following years when the only major clinical problem was recurrent leg thrombosis associated with circulating lupus anticoagulant activity, which was irregularly treated with oral anticoagulants. Renal function was always normal.

In January 1990 she voluntarily discontinued oral immunosuppressive treatment and was admitted to our hospital after a five-month history of asthenia, low grade fever and arthralgia. The patient was anuric and her serum creatinine was $308 \mu \mathrm{mol} / 1$. Complement was normal, antinuclear antibodies (ANA) were positive at a titer of $1 / 250$ and anti-DNA antibodies were $1 / 50$. Circulating cryoglobulins and immunocomplexes were negative. Three blood cultures were negative. Intravenous (iv) prednisone boluses of $120 \mathrm{mg} /$ day were administered over nine days. Cyclophosphamide (two boluses of $500 \mathrm{mg}$ each), prophylactic heparin and frusemide were also used. Acute renal failure was treated with alternative day haemodialysis.

On the 12th day she developed deep venous thrombosis in her left leg, severe Raynauld's phenomenon and progressive deterioration of consciousness and was transferred to the intensive care unit. Cerebrospinal fluid analysis and three blood cultures were negative. Cranial computed tomography (CT) was normal. Two sessions of plasmapheresis were then performed (days 15 and 17).

On day 23 of hospitalization she developed left hemipharesis with Babinski's sign, but cranial CT was unremarkable raising the suspicion of microvascular central nervous system vasculitis. During the following days, progressive dyspnoea, cyanosis, fever and bilateral diffuse alveolar infiltrates developed. On days 25 and 27 of hospitalisation we concomitantly administered further boluses of cyclophosphamide and prednisone. On day 27 assisted respiration was begun, and the patient was started on adequate doses of intravenous vancomycin, ceftazidime and gentamicin, and the cyclophosphamide and prednisone were withdrawn. During the following days, severe hypothermia, peripheral vasoconstriction, intense leukopenia and thrombopenia supervened. Several blood cultures of peripheral veins and central catheter taken on days 32 and 34 of hospitalisation were sterile. Vasoactive drugs were administered, but despite all efforts she died on 42 nd day of hospitalisation. Three blood cultures taken on day 36 yielded Cedecea neteri sp, (API System and PASCO System). The results arrived three days after she had died. The infection was resistant to amoxicillin and cephalosporins (MIC $>16 \mu \mathrm{g} / \mathrm{ml}$ for all) and also resistant to amoxicillin-clavulanic acid and aminoglycosides (MIC $8-4 \mu \mathrm{g} / \mathrm{ml}$ ), but susceptible to vancomycin (MIC $0.5 \mu \mathrm{g}$ / $\mathrm{ml}$ ) in vitro.

\section{Discussion}

Cedecea strains are rod-shaped cells conforming to the general definition of the family enterobacteriaceae. ${ }^{1}$ They are facultatively anaerobic, and lipase-, beta-galactosidase-, arginine dehydrolase-, citrate-, voges proskauer-, glucose-, mannitol-, sorbitol-, sucroseand gelatin hydrolysis-positive and deoxyribonuclease-, lysine decarboxylase-, ornithine decarboxylase-, urease-, indole-, tryptophane deaminase-, arabinose-, melibiose-, raffinose-, rhamnose- and adonitol gelatinase-negative. Some strains are negative for voges proskauer, sorbitol and sucrose reactions, and some are positive in the ornithine decarboxylate test. All strains identified have been isolated from human clinical specimens, usually from the respiratory tract. Five species of Cedecea have 


\begin{tabular}{|l|}
\hline Cedecea neteri \\
\hline - enterobacteriaceae \\
- facultative anaerobe \\
- rod-shaped \\
- causes of endocarditis \\
patient
\end{tabular}

been characterized on the basis of DNA/DNA hybridization experiments and phenotypic differences: $C$ davisae, $C$ lepage $i, C$ neteri and two unnamed ones. Only one case of human bacteraemia due to a strain of Cedecea has been reported thus far, ${ }^{2}$ in which three of four blood cultures from a patient with possible endocarditis were positive. The patient recovered after treatment. The findings supported a com-

1 Grimont PAD, Grimont F, Farmer III JJ, Asbury MA Cedecea davisae gen. nov, sp. nov. Cedecea lapagie sp. nov. new entrobacteraiaceae from clinical specimens. Int $\mathfrak{f}$ Syst Bacteriol 1981; 31: 317-26.

2 Farmer III JJ, Sheth NK, Hudzinsky JA, Rose HD, Asbury MF. Bacteremia due to Cedecea neteri sp. nov. $f$ Clin Microbiol 1982; 16: $775-8$. munity-acquired origin for this episode rather than a nosocomial infection.

Our patient suffered a fatal episode of septic shock during a severe acute flare-up of SLE including leg thrombosis, acute renal failure requiring dialysis, and respiratory failure. Although we administered high doses of immunosuppressive drugs and plasmapheresis the evolution was fatal. Hypotension may also have contributed to the acute renal failure.

Cedecea neteri appears to have been the causative agent of the final events. It was probably a nosocomial infection in an immunosuppressed patient. It is well known that patients with SLE, particularly those given long-term immunosuppressive therapy, are predisposed to severe infections., ${ }^{3,4}$ Very little is known about the role of Cedecea in human disease, as our patient illustrates. This agent may gain status as an opportunistic pathogen.

3 Hellman DB, Petri M, Whiting-O'Keefe Q. Fatal infection in systemic lupus erythematosus. The role of opportunistic pathogens. Medicine 1987; 66: 341-8.

4 Kattwinkel N, Cool L, Agnello V. Overwhelming fatal infection in a young woman after intravenous cyclosphosphamide therapy for lupus nephritis. $\mathcal{f}$ Rheumatol 1991; 18: $79-81$.

\section{Medical Anniversary WINSTON CHURCHILL, 2 MARCH 1944}

(Sir) Winston Churchill (1874-1965) addressed the Royal College of Physicians on two occasions. On this wartime day he was able to reassure the assembly that he had recovered from pneumonia due to the medical attention he had received from Lord Moran, Professor JG Scadding and Dr Evan Bedford. His second address at the Royal College of Physicians was to present his friend and physician, Lord Moran, with Moran's portrait by Pietro Annigoni. This masterly painting hangs on the ground floor of the College alongside portraits of other recent presidents. Churchill had close links with the medical profession through Moran and became honorary FRCS (1943), FRCP (1951) as well as an earlier FRS (1941). 